\title{
Transitions in density dependent harvesting of a logistic population in a slowly varying environment
}
T. Grozdanovski ${ }^{1}$
J. J. Shepherd ${ }^{2}$
A. Stacey ${ }^{3}$

(Received 3 March 2010; revised 9 June 2010)

\begin{abstract}
We previously applied a multiscale method to construct general analytic approximations to the solution of a harvested logistic system, where the system parameters vary slowly in time and the harvesting was maintained at either subcritical or supercritical levelsrepresenting survival or extinction of the population. This article extends these results by including an analytic approximation through the transition from subcritical harvesting to supercritical harvesting. This approximates the population as it is driven from a surviving population to extinction by over harvesting. These results compare favourably with numerical solutions.
\end{abstract}

\section{Contents}

\section{Introduction}

http://anziamj . austms.org. au/ojs/index.php/ANZIAMJ/article/view/2582 gives this article, (c) Austral. Mathematical Soc. 2010. Published June 23, 2010. ISSN 1446-8735. (Print two pages per sheet of paper.) 
2 Solutions away from the transition point

3 Solutions around the transition point

C398

4 Uniform approximation

C402

5 Comparison with numerical solutions

C403

6 Discussion

C404

References

C407

\section{Introduction}

Grozdanovski et al. [3] considered the evolution of a spatially homogenous single species population that was subject to harvesting at a rate proportional to the population. They showed that the change in the population over time, $p(t, \varepsilon)$, could be expressed in dimensionless form as the solution of the initial value problem

$$
\frac{d p(t, \varepsilon)}{d t}=r(\varepsilon t) p(t, \varepsilon)\left(1-\frac{p(t, \varepsilon)}{k(\varepsilon t)}\right)-\mu e(\varepsilon t) p(t, \varepsilon), \quad p\left(t=t_{i}, \varepsilon\right)=p_{i},
$$

where $\varepsilon>0$, and where $\mu>0$ and $p_{i}>0$ are dimensionless constants independent of $\varepsilon$. The initial value in equation (1) differs slightly from that of Grozdanovski et al. [3], the initial time now being arbitrary.

Grozdanovski et al. [3] concentrated on two cases: subcritical harvesting, which corresponds to $r(\varepsilon t)-\mu e(\varepsilon t)>0$ on $t \geqslant t_{i}$; and supercritical harvesting, which corresponds to $r(\varepsilon t)-\mu e(\varepsilon t)<0$ on $t \geqslant t_{i}$, where the rate of growth, $r(\varepsilon t)$, rate of harvesting, $\mu e(\varepsilon t)$, and the carrying capacity, $k(\varepsilon t)$, are assumed to be positive for all $t \geqslant t_{i}$. 
In order to simplify calculations we rewrite (1) as

$$
\frac{d p(t, \varepsilon)}{d t}=a(\varepsilon t) p(t, \varepsilon)-b(\varepsilon t) p^{2}(t, \varepsilon), \quad p\left(t=t_{i}\right)=p_{i},
$$

where $a(\varepsilon t)=(r(\varepsilon t)-\mu e(\varepsilon t))$ and $b(\varepsilon t)=r(\varepsilon t) / k(\varepsilon t)$.

Previous work [3] applied a multiscaling method to the differential equation (1) to obtain two two-term expansions representing the solutions in the subcritical and supercritical harvesting cases. These combine into one general form which, in terms of the formulation (2), becomes

$$
p(t, \varepsilon)=\frac{a\left(t_{1}\right)}{b\left(t_{1}\right)+C a\left(t_{1}\right) e^{-\sigma t_{0}}}+\varepsilon \frac{a\left(t_{1}\right) b^{\prime}\left(t_{1}\right)-b\left(t_{1}\right) a^{\prime}\left(t_{1}\right)}{a\left(t_{1}\right)\left(b\left(t_{1}\right)+C a\left(t_{1}\right) e^{-\sigma t_{0}}\right)^{2}}+\mathcal{O}\left(\varepsilon^{2}\right),
$$

where the two time variables $t_{0}$ and $t_{1}$ are

$$
t_{0}=\frac{\sigma}{\varepsilon} \int_{\varepsilon t_{i}}^{t_{1}} a(s) d s \quad \text { and } \quad t_{1}=\varepsilon t ;
$$

and $\mathrm{C}$ is a constant, determined by initial conditions, that may depend on $\varepsilon[3]$.

The subcritical harvesting case $\left(a\left(t_{1}\right)>0\right.$ on $\left.t_{1} \geqslant \varepsilon t_{i}\right)$ is obtained by setting $\sigma=1$, whereas setting $\sigma=-1$ gives the supercritical harvesting case $\left(a\left(t_{1}\right)<0\right.$ on $\left.t_{1} \geq \varepsilon t_{i}\right)$. Grozdanovski [2, Chapter 5] derived the expansion (3).

Expansion (3) displays variation of the population on two time scales: the fast time scale $t_{0}$; and the slow time scale $t_{1}$. For fixed $\varepsilon>0$ and either choice of $\sigma, t_{0} \rightarrow \infty$ as $t_{1}$ (and $\left.t\right) \rightarrow \infty$. Similarly for $t_{1}$ bounded away from $\varepsilon t_{i}$ as $\varepsilon \rightarrow 0, t_{0} \rightarrow \infty$ as $\varepsilon \rightarrow 0$. Thus, on any set of $t_{1}$ values bounded away from $\varepsilon t_{i}$ as $\varepsilon \rightarrow 0$, the expansion (3) passes through a rapid transient state (via fast $t_{0}$ variation) to a slowly varying limiting state,

$$
p(t, \varepsilon)=\frac{a\left(t_{1}\right)}{b\left(t_{1}\right)}-\varepsilon \frac{a^{\prime}\left(t_{1}\right) b\left(t_{1}\right)-b^{\prime}\left(t_{1}\right) a\left(t_{1}\right)}{a\left(t_{1}\right) b\left(t_{1}\right)^{2}}+\cdots,
$$


when $\sigma=1$, and the zero state when $\sigma=-1$. Both these states are independent of $\mathrm{C}$, and hence independent of any given initial conditions.

Further observation of the expansion (3) shows that, in general, as $a\left(t_{1}\right) \rightarrow 0$ the first term tends to zero while the second term becomes unbounded. This implies that in any region where $a\left(t_{1}\right)=\mathcal{O}(\varepsilon)$, there is a disordering of the two terms in (3), leading to the failure of this expansion to represent the solution of (2). This was also noted by Grozdanovski et al. [3]. In particular the two term expansion (3) fails in any region that includes a zero of $a\left(t_{1}\right)$.

This article extends earlier work [3] by considering the case of a system that starts in a state of subcritical harvesting and at some point in time moves to a state of supercritical harvesting, where it remains. The change point is a zero of $a\left(t_{1}\right)$, which we term a transition point. By the discussion above the expansion (3) fails (is disordered) in a neighbourhood of this transition point. Such failure can occur naturally when the rate of harvesting increases over time, or environmental factors result in $r\left(t_{1}\right)$ declining over time, causing $r\left(t_{1}\right)-\mu e\left(t_{1}\right)$ to change sign.

More specifically we assume that the function $a\left(t_{1}\right)$ has a single isolated simple zero, at a point we denote by $\overline{\mathrm{t}}_{1}=\varepsilon \overline{\mathrm{t}}$ (equivalently $\overline{\mathrm{t}}=\overline{\mathrm{t}}_{1} / \varepsilon$ ); that is

$$
a\left(\bar{t}_{1}\right)=0 \quad \text { and } \quad a^{\prime}\left(\bar{t}_{1}\right)<0 .
$$

We also assume that the transition point $\bar{t}$ occurs well after the the initial transient behavior of the system has died away and that there are no extended regions where $a\left(t_{1}\right)=\mathcal{O}(\varepsilon)$.

In dealing with this transition in the following sections, we consider three $t_{1}$ time regions as follows. We choose $\delta$ to be small, positive and independent of $\varepsilon$. Then Regions 1, 2 and 3 are defined by: Region $1, \varepsilon t_{i} \leqslant t_{1} \leqslant \bar{t}_{1}-\delta$; Region $2, \overline{\mathrm{t}}_{1}-\delta \leqslant \mathrm{t}_{1} \leqslant \overline{\mathrm{t}}_{1}+\delta$; Region $3, \overline{\mathrm{t}}_{1}+\delta \leqslant \mathrm{t}_{1}<\infty$.

We approximate solutions to the differential equation of (2) in Region 1 and 3 using expansions of the type (3), as constructed by Grozdanovski et al. [3]. We construct an approximate solution in the (small) transition Region 2 
using local analysis. This solution links to the expansions of Regions 1 and 3 using a matching technique, to provide an approximation to the evolving population on all of $t \geqslant t_{i}$.

\section{Solutions away from the transition point}

Here we consider the solutions of the differential equation of (2) in Regions 1 and 3 ; that is, away from the transition region.

As noted above, we here only consider situations where the system makes a single isolated transition from subcritical harvesting to supercritical harvesting, corresponding to a zero of $a\left(t_{1}\right)=r\left(t_{1}\right)-\mu e\left(t_{1}\right)$.

Since $a\left(t_{1}\right)>0$ and is independent of $\varepsilon$ on all of Region 1, the expansion (3) with $\sigma=1$ represents the population there, and application of the initial conditions in (2) [2] gives the two term expansion in Region 1 as

$$
\begin{aligned}
p(t, \varepsilon)= & \frac{a\left(t_{1}\right) a_{i} p_{i}}{b\left(t_{1}\right) a_{i} p_{i}+a\left(t_{1}\right)\left(a_{i}-b_{i} p_{i}\right) e^{-t_{0}}} \\
& -\varepsilon \frac{p_{i}^{2}\left[a_{i}^{3}\left(a^{\prime}\left(t_{1}\right) b\left(t_{1}\right)-b^{\prime}\left(t_{1}\right) a\left(t_{1}\right)\right)+a^{3}\left(t_{1}\right)\left(b_{i}^{\prime} a_{i}-a_{i}^{\prime} b_{i}\right) e^{-t_{0}}\right]}{a\left(t_{1}\right) a_{i}\left[b\left(t_{1}\right) a_{i} p_{i}+a\left(t_{1}\right)\left(a_{i}-b_{i} p_{i}\right) e^{-t_{0}}\right]^{2}} \\
& +\mathcal{O}\left(\varepsilon^{2}\right),
\end{aligned}
$$

where $t_{0}$ and $t_{1}$ are given by (4) with $\sigma=1$, and the subscript $i$ denotes values taken at the initial time, $t=t_{i}$.

In Region $3, a\left(t_{1}\right)<0$, so the expansion (3) with $\sigma=-1$ applies. However, $t_{1}=\varepsilon t_{i}$ is not an initial point for Region 3 , so we modify the definition of (4) to be

$$
\tilde{t}_{0}=-\frac{1}{\varepsilon} \int_{\bar{t}_{1}}^{t_{1}} a(s) d s \text { and } \quad t_{1}=\varepsilon t
$$


Then our expansion in Region 3 becomes

$$
p(t, \varepsilon)=\frac{a\left(t_{1}\right)}{b\left(t_{1}\right)+C a\left(t_{1}\right) e^{\tilde{t}_{0}}}+\varepsilon \frac{a\left(t_{1}\right) b^{\prime}\left(t_{1}\right)-b\left(t_{1}\right) a^{\prime}\left(t_{1}\right)}{a\left(t_{1}\right)\left(b\left(t_{1}\right)+C a\left(t_{1}\right) e^{\tilde{t}_{0}}\right)^{2}}+\mathcal{O}\left(\varepsilon^{2}\right) .
$$

This leaves the constant $\mathbf{C}$ in (9) undetermined. We return to this in the next section.

\section{Solutions around the transition point}

We now turn to solutions of the differential equation in (2) in Region 2. As noted above, when $a\left(t_{1}\right)=\mathcal{O}(\varepsilon)$ the expansion $(7)$ becomes disordered and fails to represent the solution of (2). Under our assumptions above, and noting (6), we see that $a\left(t_{1}\right)=\mathcal{O}(\varepsilon)$ corresponds to an interval of extent $\mathcal{O}(\varepsilon)$ centred on $\bar{t}_{1}$, the zero of $a\left(t_{1}\right)$.

We now examine solutions of the differential equation (2) in a small interval about the transition point $\overline{\mathrm{t}}_{1}$ that contains the interval above. To do this we consider a new time variable $\tau$,

$$
\mathrm{t}_{1}=\overline{\mathrm{t}}_{1}+\varepsilon^{\alpha} \tau
$$

where exponent $\alpha$ is a positive constant to be determined, and define the solution of (2) about $\bar{t}_{1}$ by

$$
\tilde{p}(\tau, \varepsilon) \equiv p\left(\bar{t}_{1}+\varepsilon^{\alpha} \tau, \varepsilon\right) .
$$

For bounded $\tau$ values, (10) describes an interval centred on $t_{1}=\bar{t}_{1}$ of extent $\mathcal{O}\left(\varepsilon^{\alpha}\right)$.

In terms of these variables, the differential equation of (2) becomes

$$
\frac{d \tilde{p}}{d \tau}=a\left(\bar{t}_{1}+\varepsilon^{\alpha} \tau\right) \varepsilon^{\alpha-1} \tilde{p}-b\left(\bar{t}_{1}+\varepsilon^{\alpha} \tau\right) \varepsilon^{\alpha-1} \tilde{p}^{2} .
$$


Under our earlier assumptions,

$$
\mathrm{a}\left(\overline{\mathrm{t}}_{1}+\varepsilon^{\alpha} \tau\right)=\mathcal{O}\left(\varepsilon^{\alpha}\right)
$$

for bounded $\tau$ values. Thus the first term on the right side of $(12)$ is $\mathcal{O}\left(\varepsilon^{2 \alpha-1}\right)$; whereas the second term is $\mathcal{O}\left(\varepsilon^{\alpha-1}\right)$.

We now choose an exponent $\alpha$ that allows (12) to generate solutions that display the necessary limiting properties as $\tau \rightarrow \pm \infty$, to allow a matching procedure to be used.

Under (13), the first term on the right side of (12) will show an order balance with the left side if we choose $\alpha=1 / 2$. However, the second right side term is now unbounded as $\varepsilon \rightarrow 0$, so overall balancing fails. Similarly, choosing $\alpha=1$ balances the left side and the second right side term, but leaves the first right side term as $\mathcal{O}(\varepsilon)$. Thus the dominant differential equation in our $\mathcal{O}\left(\varepsilon^{\alpha}\right)$ region is of the form

$$
\frac{d \tilde{p}}{d \tau}=(\text { constant }) \tilde{p}^{2}
$$

which does not have solutions displaying the desired limiting properties as $\tau \rightarrow \pm \infty$. Again balancing does not succeed.

In order to gain further insight, we look at the two term expansion (7) about $\bar{t}_{1}$. In Region 1 , at points well after the transients have died out, the solution (7) takes the form (5). Substituting for $\boldsymbol{t}_{1}$ from (10) and expanding for small $\varepsilon$ gives (5) as

$$
\tilde{p}(\tau, \varepsilon)=\varepsilon^{1 / 2}\left(\frac{a_{1}^{\prime} \tau}{b_{1}}-\frac{1}{b_{1} \tau}\right)+\cdots
$$

where the subscript 1 denotes values at $t_{1}=\bar{t}_{1}$. This suggests that for small $\varepsilon$ the solution around the transition point has the form

$$
\tilde{p}(\tau, \varepsilon)=\varepsilon^{1 / 2} w(\tau),
$$


and the equation (12) becomes

$$
\frac{\mathrm{d} w}{\mathrm{~d} \tau}=\mathrm{a}\left(\overline{\mathrm{t}}_{1}+\varepsilon^{\alpha} \tau\right) \varepsilon^{\alpha-1} w-\mathrm{b}\left(\overline{\mathrm{t}}_{1}+\varepsilon^{\alpha} \tau\right) \varepsilon^{\alpha-1 / 2} w^{2} .
$$

Noting (13), we see that now the first term on the right side of $(17)$ is $\mathcal{O}\left(\varepsilon^{2 \alpha-1}\right)$, as before, but now the second term is $\mathcal{O}\left(\varepsilon^{\alpha-1 / 2}\right)$. Thus an order balance is achieved on both sides if we choose $\alpha=1 / 2$. Substituting $\alpha=1 / 2$ and

$$
w(\tau, \varepsilon)=w_{0}(\tau)+\varepsilon^{1 / 2} w_{1}(\tau)+\cdots
$$

into (17), and taking leading order terms gives

$$
\frac{d w_{0}}{d \tau}=a_{1}^{\prime} \tau w_{0}-b_{1} w_{0}^{2}
$$

which has solution

$$
w_{0}(\tau)=\frac{2 \sqrt{-a_{1}^{\prime}} \exp \left(\frac{1}{2} a_{1}^{\prime} \tau^{2}\right)}{b_{1} \sqrt{2 \pi} \operatorname{erf}\left(\frac{1}{2} \sqrt{-2 a_{1}^{\prime}} \tau\right)+D},
$$

where

$$
\operatorname{erf}(x)=\frac{2}{\sqrt{\pi}} \int_{0}^{x} \exp \left(-t^{2}\right) d t
$$

and D is an arbitrary constant.

Now consider the consequences of these calculations. We have $\varepsilon^{1 / 2} w_{0}(\tau)$, with $\boldsymbol{w}_{0}(\tau)$ given by $(20)$, as a leading order approximation to $\tilde{p}(\tau, \varepsilon)$ in a region of extent $\mathcal{O}\left(\varepsilon^{1 / 2}\right)$ about the transition point. At points $t_{1}<\bar{t}_{1}$, the expansion (5) represents the solution to leading order. In terms of the local variable $\tau$ this becomes (15). As they stand, these two regions remain separated as $\varepsilon \rightarrow 0$. However, if there is an overlap interval, common to both as $\varepsilon \rightarrow 0$, we claim that both expansions are valid in this overlap region. This leads to the simple matching condition that as $\tau \rightarrow-\infty, \varepsilon^{1 / 2} \mathcal{W}_{0}(\tau)$ must tend to the expression (15). 
Calculating the expansion of (20) as $\tau \rightarrow-\infty$, and comparing with (15) shows that this is only possible if we set $D=b_{1} \sqrt{2 \pi}$, making the leading order approximation to the solution of (2) about $\overline{\boldsymbol{t}}_{1}$

$$
\tilde{p}(\tau, \varepsilon)=\varepsilon^{1 / 2} \omega(\tau)=\varepsilon^{1 / 2} \frac{2 \sqrt{-a_{1}^{\prime}} \exp \left(\frac{1}{2} a_{1}^{\prime} \tau^{2}\right)}{b_{1} \sqrt{2 \pi}\left[1+\operatorname{erf}\left(\frac{1}{2} \sqrt{-2 a_{1}^{\prime}} \tau\right)\right]}+\cdots .
$$

This choice for the constant $\mathrm{D}$ is confirmed when we note that the asymptotic expansion of (22), as $\tau \rightarrow-\infty$, is

$$
\tilde{p}(\tau, \varepsilon)=\varepsilon^{1 / 2}\left[\frac{a_{1}^{\prime} \tau}{b_{1}}-\frac{1}{b_{1} \tau}+\mathcal{O}\left(\frac{1}{\tau^{3}}\right)\right] .
$$

We now consider the matching of the expansion (9) in Region 3 to that of Region 2, (22). If we assume a set of $\tau$ values that overlap Regions 2 and 3 , these are characterized by large $\tau$. For $\tau \rightarrow \infty$, (22) tends to

$$
\frac{\sqrt{-a_{1}^{\prime}}}{b_{1} \sqrt{2 \pi}} \exp \left(\frac{1}{2} a_{1}^{\prime} \tau^{2}\right)
$$

Now consider the expansion (9) in an overlap region. Since $t_{1}=\bar{t}_{1}+\varepsilon^{1 / 2} \tau$, we have that $\mathbf{a}\left(\mathbf{t}_{1}\right)=\mathcal{O}\left(\varepsilon^{1 / 2}\right)$ in such a region, and the first term of (9) dominates, hence there is no disordering.

To leading order in small $\varepsilon$,

$$
\tilde{t}_{0}=-\frac{1}{2} a_{1}^{\prime} \tau^{2}
$$

and so the leading term of (9) becomes, to leading order, after some rearranging,

$$
\frac{\varepsilon^{1 / 2} a_{1}^{\prime} \tau \exp \left(\frac{1}{2} a_{1}^{\prime} \tau^{2}\right)}{b_{1} \exp \left(\frac{1}{2} a_{1}^{\prime} \tau^{2}\right)+C \varepsilon^{1 / 2} a_{1}^{\prime} \tau} .
$$


Now, in any proposed overlap region between Regions 2 and $3, \tau$ is large and positive so it is reasonable to ignore the exponential in the denominator of (26). Hence (24) and (26) are matched by choosing

$$
C=\frac{b_{1} \sqrt{2 \pi}}{\varepsilon^{1 / 2} \sqrt{-a_{1}^{\prime}}} .
$$

With this value of $\mathbf{C}$, to leading order, (9) becomes

$$
\begin{aligned}
p(t, \varepsilon) & =\frac{\varepsilon^{1 / 2} a\left(t_{1}\right)}{\varepsilon^{1 / 2} b\left(t_{1}\right)+\left(b_{1} \sqrt{2 \pi} / \sqrt{-a_{1}^{\prime}}\right) a\left(t_{1}\right) e^{\tilde{t}_{0}}} \\
& =\frac{\varepsilon^{1 / 2} \sqrt{-a_{1}^{\prime}}}{b_{1} \sqrt{2 \pi}} e^{-\tilde{t}_{0}}+\mathcal{O}\left(\varepsilon^{1 / 2}\right) .
\end{aligned}
$$

In view of (25), equation (28) is the same, to leading order, as the transition solution (22); that is, the expansion away from the transition in Region 3 is effectively zero, and (22) represents the solution from $t_{1}=\bar{t}_{1}$ onwards. This feature is used in the following section.

\section{Uniform approximation}

Having found a solution about the transition point $\bar{t}_{1}$ it is straightforward to construct a uniform solution in the interval $\left[t_{i}, \bar{t}\right]$. We do this by additive composition; that is, we add the two solutions given by (22) and (3) and subtract off the common part. The common part is just the expression given by (15). Using (4) and (10) we rewrite $\tau$ in terms of $t$ and $\bar{t}$ as $\tau=\varepsilon^{1 / 2}(t-\bar{t})$. The common part is now

$$
c p=\varepsilon^{1 / 2}\left(\frac{a_{1}^{\prime} \varepsilon^{1 / 2}(t-\bar{t})}{b_{1}}-\frac{1}{b_{1} \varepsilon^{1 / 2}(t-\bar{t})}\right),
$$


where $\bar{t}$ is the transition point. The uniform approximation valid for the interval $\left[t_{i}, \bar{t}\right]$ is then

$$
\begin{aligned}
& p(t, \varepsilon)=\frac{a(\varepsilon t) a_{i} p_{i}}{b(\varepsilon t) a_{i} p_{i}+a(\varepsilon t)\left(a_{i}-b_{i} p_{i}\right) e^{-t_{0}}} \\
& -\varepsilon \frac{p_{i}^{2}\left\{a_{i}^{3}\left[a^{\prime}(\varepsilon t) b(\varepsilon t)-b^{\prime}(\varepsilon t) a(\varepsilon t)\right]+a^{3}(\varepsilon t)\left[b_{i}^{\prime} a_{i}-a_{i}^{\prime} b_{i}\right] e^{-t_{0}}\right\}}{a(\varepsilon t) a_{i}\left[b(\varepsilon t) a_{i} p_{i}+\left(a(\varepsilon t) a_{i}-a(\varepsilon t) b_{i} p_{i}\right) e^{-t_{0}}\right]^{2}} \\
& +\varepsilon^{1 / 2} \frac{2 \sqrt{-a_{1}^{\prime}} \exp \left(\frac{1}{2} a_{1}^{\prime} \varepsilon(t-\bar{t})^{2}\right)}{b_{1} \sqrt{2 \pi}\left[1+\operatorname{erf}\left(\frac{1}{2} \sqrt{-2 a_{1}^{\prime}} \varepsilon^{1 / 2}(t-\bar{t})\right)\right]} \\
& -\varepsilon^{1 / 2}\left(\frac{a_{1}^{\prime} \varepsilon^{1 / 2}(t-\bar{t})}{b_{1}}-\frac{1}{b_{1} \varepsilon^{1 / 2}(t-\bar{t})}\right)+\mathcal{O}\left(\varepsilon^{2}\right)
\end{aligned}
$$

where $t_{0}$ is given by (4) with $\sigma=1$.

As argued in the previous section, the transition expansion (22) is used to represent the population throughout all of $[\bar{t}, \infty)$, to leading order. Written in terms of $t$, this is

$$
\tilde{p}(t, \varepsilon)=\varepsilon^{1 / 2} \frac{2 \sqrt{-a_{1}^{\prime}} \exp \left(\frac{1}{2} a_{1}^{\prime} \varepsilon(t-\bar{t})^{2}\right)}{b_{1} \sqrt{2 \pi}\left[1+\operatorname{erf}\left(\frac{1}{2} \sqrt{-2 a_{1}^{\prime}} \varepsilon^{1 / 2}(t-\bar{t})\right)\right]}+\cdots .
$$

The additive composition as used for Regions 1 and 2 could be used for this. The common part between Regions 2 and 3 is zero. Moreover, since all terms of (9) are exponentially small as $\varepsilon \rightarrow 0$ in Region 2 , the expansion there is effectively the zero expansion. So composition between Regions 2 and 3 gives the result (31).

\section{Comparison with numerical solutions}

Here we compare the analytic approximation, given by (30) to the left of the transition point, and (22) to the right of the transition point, with the 
numerical solution of (2). We first consider the case where the carrying capacity, $k\left(t_{1}\right)=1$, is a constant while the growth rate, $r\left(t_{1}\right)$, and harvesting rate, $e\left(t_{1}\right)$, both vary slowly:

$$
k\left(t_{1}\right)=1, \quad r\left(t_{1}\right)=0.8+0.02 \sin \left(t_{1}\right), \quad e\left(t_{1}\right)=e^{0.01 t_{1}}-0.32,
$$

with $\mu=1, t_{i}=0, \varepsilon=0.01$ and $p_{i}=0.005$. Figure 1 shows that the analytic approximation gives good agreement with the numerical solution, almost coinciding.

In this next example we consider almost the same system:

$$
k\left(t_{1}\right)=1, \quad r\left(t_{1}\right)=0.8+0.02 \sin \left(t_{1}\right), \quad e\left(t_{1}\right)=e^{0.007 t_{1}}-0.27,
$$

with $\mu=1, t_{i}=0, \varepsilon=0.01$ and $p_{i}=0.005$. The slight change in the harvesting rate, $e\left(t_{1}\right)$, has been chosen so that the difference $a\left(t_{1}\right)$ approaches $1.5 \varepsilon$ in the vicinity of $t=500$. Under these conditions we expect the two term approximation, given by (30), to begin to fail. Figure 2 shows this failure where the analytic approximation deviates from the numerical solution centred around $t=550$.

\section{Discussion}

For small $\varepsilon>0$, expansions (30) and (31) provide simple explicit leading order approximations to the evolving population $p(t, \varepsilon)$ given by $(2)$ when we move through a transition from subcritical to supercritical harvesting, as discussed in Section 1. When compared with the results of numerical calculation, they give excellent agreement under suitable conditions.

These approximations are general in form, and may be applied to any arbitrary combination of $a(\varepsilon t)$ and $b(\varepsilon t)$ (or $r(\varepsilon t), e(\varepsilon t)$ and $k(\varepsilon t)$ ) satisfying simple smoothness conditions and displaying a single transition at $t=\bar{t}$ satisfying (6). 


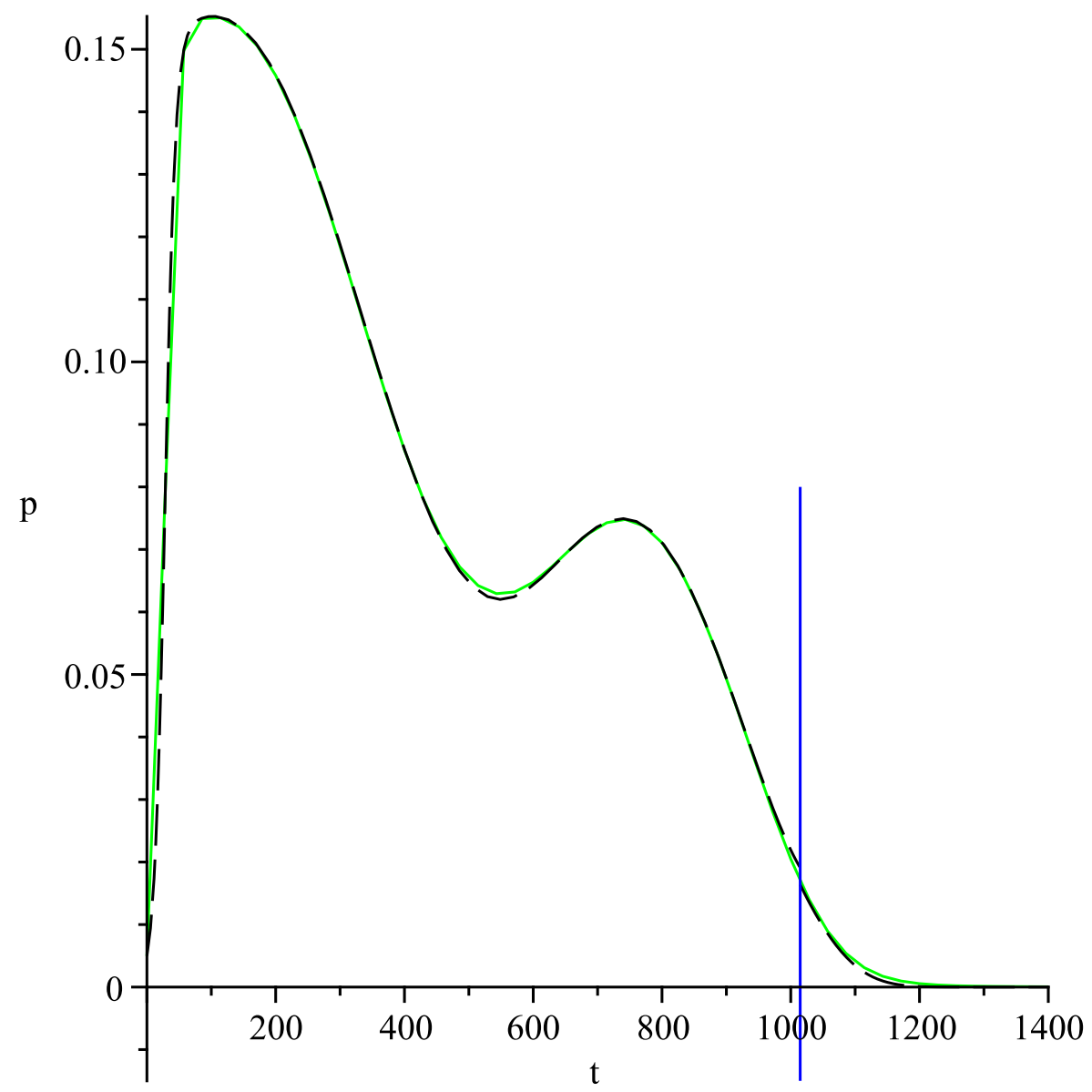

Figure 1: Population, $p$, as a function of time, $t$, for harvesting, where the multi-scale approximation (using (30) to the left of the transition point and (22) to the right of the transition point) is shown as black-dashed and numerical solution as green-solid; for the choice of system equations (32), with $p_{0}=0.005$ and $\varepsilon=0.01$. The black dashed curve sits almost exactly on top of the green continuous curve. The blue vertical line identifies the time at which the transition occurs. 


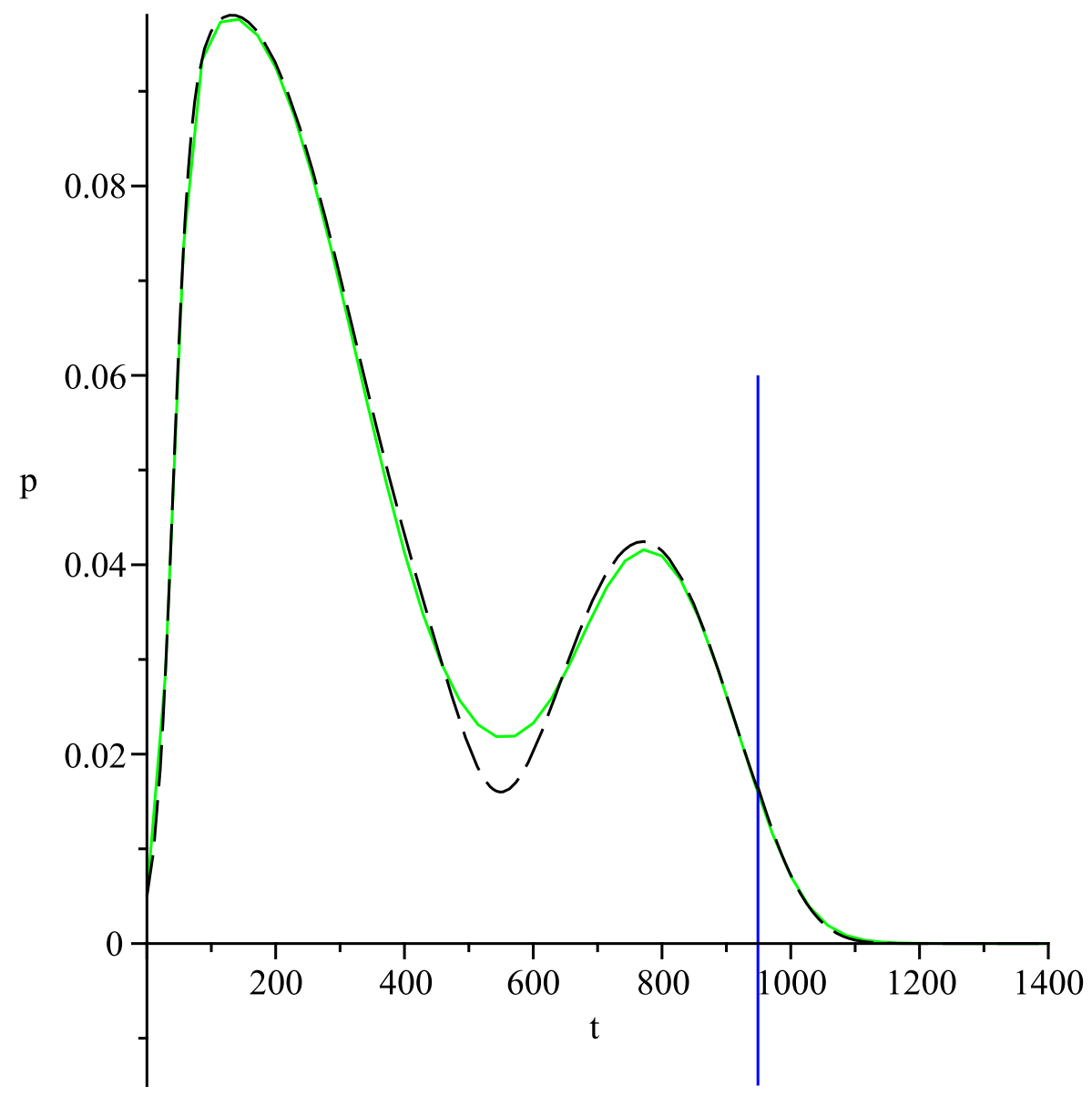

Figure 2: Population, $p$, as a function of time, $t$, for harvesting, where the multi-scale approximation (using (30) to the left of the transition point and (22) to the right of the transition point) is shown as black-dashed and numerical solution as green-solid; for the choice of system equations (33), with $p_{0}=0.005$ and $\varepsilon=0.01$. The analytic approximation (black dashed curve) differs from the numerical solution (green continuous curve) in the region around $t=500$, which correspond to the situation where the two functions $r\left(t_{1}\right)$ and $e\left(t_{1}\right)$ are close together but do not cross. The blue vertical line identifies the time at which the transition occurs. 
It is interesting to compare the problem (2) with the particular example of Dahlquist's Knee [1, 4]

$$
\varepsilon \frac{d y}{d t}=(1-t) y-y^{2}, \quad y(0, \varepsilon)=y_{0} .
$$

Replacing $\mathrm{t}$ by $\mathbf{t} / \varepsilon$ in (34), we have

$$
\frac{d y}{d t}=(1-\varepsilon t) y-y^{2}, \quad y(0, \varepsilon)=y_{0} .
$$

This is now of the form of (2), with $a(\varepsilon t)=1-\varepsilon t, b(\varepsilon t)=1$ and displays a transition at $t_{1}=\varepsilon t=1$. It has been used [1] to demonstrate the failure of numerical schemes to solve such problems when $\varepsilon$ is small and positive. It may be analyzed using matching techniques [4], by locating an initial layer at $t_{1}=0$ of thickness $\mathcal{O}(\varepsilon)$ and a transition layer at $t_{1}=1$ of thickness $\mathcal{O}(\sqrt{\varepsilon})$, then matching between the various subregions of $t_{1} \geqslant 0$.

Our expansion (30) combines rapidly changing transient components, arising from the $t_{0}$ variation adjacent to $t_{1}=\varepsilon t_{i}$, with a slowly varying component (5) outside this initial region. These correspond to the initial layer and 'outer expansion' arising in the matching analysis of O'Malley [4]. Our layer analysis at the transition point parallels previous work [4], while O'Malley [4] identifies the 'outer expansion' beyond the transition point $t_{1}=1$ as the zero expansion (compare with our Section 6 discussion).

\section{References}

[1] G. Dahlquist and G. Soderlind. Some problems related to stiff nonlinear systems. In R. Glowinski and J. L. Lions, editors, Computing Methods in Applied Sciences and Engineering V, pages 57-74. North-Holland, Amsterdam, 1982. C407

[2] T. Grozdanovski. Multi-Scaling Methods Applied to Population Models. Ph.D. Thesis, RMIT University, 2009. C395, C397 
[3] T. Grozdanovski, J. J. Shepherd and A. Stacey. Density dependent harvesting of a logistic population in a slowly varying environment. ANZIAM J. 51:C32-C47, 2010. http://anziamj.austms.org.au/ ojs/index.php/ANZIAMJ/article/view/2271 C394, C395, C396

[4] R. E. O'Malley Jr. Singular Perturbation Methods for Ordinary Differential Equations. Springer-Verlag, New York, 1991. C407

\section{Author addresses}

1. T. Grozdanovski, School of Mathematical and Geospatial Sciences, RMIT University, Melbourne, 3001, Australia.

mailto:e61565@ems.rmit.edu.au

2. J. J. Shepherd, School of Mathematical and Geospatial Sciences, RMIT University, Melbourne, 3001, Australia. mailto: jshep@rmit.edu.au

3. A. Stacey, School of Mathematical and Geospatial Sciences, RMIT University, Melbourne, 3001, Australia. mailto:stacey@rmit.edu.au 